\title{
DE CAMINO A LA ESCENA: TRADUCCIÓN, TRANSFERENCIA DE GÉNEROS Y LA FORMACIÓN LITERARIA DE CHRISTOPHER MARLOWE
}

\author{
José María PÉREZ FERNÁNDEZ
}

Universidad de Granada

El teatro isabelino emerge de una amalgama de fragmentos de diferentes géneros. En el nuevo drama que surge en Inglaterra entre comienzos del siglo XVI y mediados del XVII confluyen convenciones y discursos, personajes y estilos de la más variada procedencia: el teatro religioso tardío-medieval, tanto popular y semi-bufo como serio y más ortodoxo, el nuevo teatro humanista salido de las universidades, de un elevado carácter retórico y encauzado a través de la tradición senequista y terenciana, con una fuerte base en los ejercicios de debate que los estudiantes llevan a cabo, y en general la tradición clásica en su sentido más amplio, incluidos géneros no necesariamente dramáticos.

En este artículo me propongo explorar el papel que la traducción jugó en la formación literaria de Christopher Marlowe como proceso que le permitió asimilar diferentes géneros y discursos previos en una práctica que desembocaría en sus obras dramáticas más importantes. Así, antes de lanzarse a su carrera de dramaturgo, y de la producción de sus obras, construidas sobre su famosa "mighty line", Marlowe llevó a cabo una traducción de la Farsalia de Lucano, en la que exploró las posibilidades estilísticas del género épico, y adquirió la capacidad para componer extensos párrafos versuales en los que articular sus monólogos dramáticos. La Farsalia dio a Marlowe la oportunidad de experimentar con el decasílabo blanco y explotar sus posibilidades frente a otros metros. Esta experimentación convirtió a Marlowe en uno de los primeros autores en desarrollar con éxito el decasílabo yámbico sin rima que se convertiría en la piedra angular del teatro isabelino, sobre todo en la obra de Shakespeare. Con posterioridad, además, este decasílabo blanco habría de convertirse en el metro canónico de la tradición poética en lengua inglesa.

Pero antes de modular el decasílabo blanco y de situarse en la base de una tradición de siglos Marlowe también tomó otros caminos durante la primera parte de su breve carrera literaria en su afán por explorar las posibilidades estilísticas de su idioma. Me refiero en concreto a la primera traducción conocida de Marlowe, la de los Amores de Ovidio. En esta traducción Marlowe no ha adoptado todavía el verso blanco, aunque ya hace uso de decasílabos pareados. Se trata de una traducción tentativa, en la que a veces el texto sufre de cierto encorsetamiento en términos sintácticos que era muy típico de la época y que se caracteriza a grandes rasgos por la regularidad en la colocación de la cesura tras la cuarta o la sexta sílaba, y la insistencia en hacer coincidir el final de los versos con el límite de periodos sintácticos de orden superior ${ }^{1}$. Una regularidad que se ve reforzada por la rima. El resultado es una suce-

\footnotetext{
${ }^{1}$ Sobre los precedentes de finales del XVI, y un cuidado análisis y evaluación del desarrollo del estilo métrico a lo largo de este siglo, véanse los capítulos 3 y 4 de Susanne Woods (pp. 69-128), donde se trata, entre otras, de la cuestión de la regularidad métrica y el desarrollo de nuevas formas métricas así como de la renovación de anteriores. En general, el libro de la profesora Woods constituye un excelente punto de partida para evaluar la situación de la que partían las formas versuales inglesas en tiempos de Christopher Marlowe.
} 
sión de versos con una sintaxis algo envarada y de corto alcance, en la que el estilo avanza a golpes bruscos, de medio verso en medio verso y sin la fluidez que proporcionan periodos sintácticos más amplios y mejor articulados ${ }^{2}$. Se puede apreciar un ejemplo de este encorsetamiento en la segunda elegía del primer libro, versos 21-28:

What needst thou warre, I sue to thee for grace,

With armes to conquer armelesse men is base.

Yoake Venus Doves, put Mirtle on thy haire,

Vulcan will give thee chariots rich and faire.

The people thee applauding thou shalt stand,

Guiding the harmeless Pigeons with thy hand.

Yong men, and women shalt thou lead as thrall,

So will thy triumph seeme magnificall.

\section{(I.2, Roma Gill, ed. pp.14-15)}

En otros poemas, por otra parte, ya se empieza a vislumbrar algo de la soltura estilística que Marlowe estaba adaptando del modelo de flexibilidad de la lengua latina, y que desarrollaría de forma más arrolladora en la traducción de Lucano. Compárense los anteriores con los siguientes ocho versos, que sostienen dos periodos de cuatro versos cada uno, con el stacatto sintáctico y rítmico del párrafo anterior:

Feardst thou to dresse them being fine and thinne

Like to the silke the curious Seres spinne,

Or thrids which spiders slender foote drawes out

Fastning her light web some old beame about.

Not black, nor golden were they to our viewe,

Yet although neither mixt of eithers hue,

Such as in hilly Idas watry plaines,

The Cedar tall spoyld of his barke retaines.

$$
\text { (I.14, Roma Gill, ed. pp.32-33) }
$$

Además de constituir un excelente ejemplo en términos de estilo a imitar y reproducir en inglés, los Amores de Ovidio presentan interesantes muestras de estrategias estilísticas que implican directamente la elección de géneros y los estilos asociados a cada uno de ellos. De estas estrategias Marlowe se apropia de manera muy provechosa, sobre todo si se contemplan a la luz de su producción posterior.

Éste el caso de la disculpa que ofrece Ovidio en el poema que abre los Amores por haber descendido de género y tema, y escribir del amor en lugar de la guerra: en otras palabras, por haberse decantado por la lírica amorosa en lugar de la poesía épica. Con la elección de Ovidio como su primera traducción, Marlowe emprende una carrera literaria que ha de llevarle posteriormente a otra traducción del género épico,

\footnotetext{
${ }^{2}$ Véase Preminger \& Brogan 1993, p. 522: "The transformation of the Chaucerian line into what Puttenham and Dryden called the 'heroic' line was evolutionary, spanning the 16th c. from ca. 1520 to $1600 \ldots$ But the imposition of the hierarchy of pauses on the loose couplet inherited from Chaucer was more dramatic, taking place almost explosively between 1590 and 1600, when numbers of English poets translated and adapted Latin poems in elegiac distichs... achieving or approximating a correspondence of couplet to distich... The immediate result of this structural imitation was the affixing onto English couplets of the Latin distich's hierarchy of pauses and of a correspondingly balanced rhetoric... English rhyme, which had the same closural power as the recurrent half-line pattern in the pentameter of the Latin distich, allowed the English measure, once it outgrew its dependence on the Latin model, to become much more flexible and thus absorb the modifications by which successive English poets from Donne to Crabbe transformed it into a medium of great expressive power."
} 
y finalmente a culminar este aprendizaje literario en un estilo resultante de la integración de diversos fragmentos y técnicas en los que se ha curtido con anterioridad. Bajo esta perspectiva, resulta esclarecedor explorar la actitud de Marlowe hacia el estilo y el género épico, en particular teniendo en cuenta cómo tuvo que abordar en esta primera traducción de Ovidio una cierta actitud anti-épica.

En los primeros versos, Marlowe encontró una serie de afirmaciones, no exentas de ironía, en las que Ovidio declara su intención de dedicarse al género épico, o sea, a lo que cualquier autor con ambición de fama y prestigio había de hacer si su intención era seguir el ejemplo de los más altos modelos precedentes:

With Muse upreard I meant to sing of Armes,

Choosing a subject fit for fierce alarmes.

(I.1, vv. 5-6, Gill, ed. p. 13)

Arma gravi numero violentaque bella parabam

edere, materia conveniente modis.

(Amores, I.1-2)

Esta significativa referencia inicial a las armas (arma es la palabra usada por el poeta) con la que Ovidio abre el primer verso predispone al lector a un encuentro directo con el género épico a través del eco de lo que ya entonces era un clásico reciente: la Eneida de Virgilio. El tono de los Amores queda así establecido desde el comienzo en toda la complejidad y versatilidad de registros y fugaces incursiones en géneros ajenos que Ovidio irá poniendo ante la mirada del lector sólo para ser inmediatamente rechazados o de alguna manera subvertidos. La inteligente y sutil irreverencia de la obra de Ovidio queda demostrada tras esta referencia directa al maestro, a la creciente sombra del padre de la épica latina puesta al servicio del imperio de Augusto, para a continuación renunciar al género épico y su estilo. Nótese cómo en estos dos primeros versos están condensados los temas y la doctrina de los géneros y del decoro, así como de la forma poética asociada a ellos. El tema aparece nombrado en arma y violentaque bella, la forma poética -esto es, el metro- en gravi numero, y finalmente la adecuación de tema y modo en materia conveniente modis. Cuesta imaginar una exposición más breve, correcta y ajustada de las premisas de esta ortodoxa doctrina. Siguen a estos versos una serie de consideraciones que vienen a dar cuerpo y sustancia verbal a la noción de decorum, en la tradición horaciana de adecuación de géneros y estilos, y el prestigio asociado a cada uno de ellos. Declara el poeta, dirigiéndose a Cupido, en la versión de Marlowe:

We are the Muses Prophets, none of thine.

What if thy mother take Dianas bowe?

Shall Dian fanne, when loue begins to glowe.

In woodie groves ist meete that Ceres raigne?

And quiver-bearing Dian till the plaine.

Who'le set the faire trest sunne in battle ray

While Mars doth take the Aonian Harpe to play.

Great are thy kingdomes, over strong and large,

Ambitious impe, why seekst thou further charge?

$$
\text { (I.1. vv. 10-18; Gill, ed. p. 13) }
$$


'Quis tibi, saeve puer, dedit hoc in carmina iuris?

Pieridum vates, non tua turba sumus.

quid, si praeripiat flavae Venus arma Minervae,

ventilet accensas flava Minerva faces?

quis probet in silvis Cererem regnare iugosis,

lege pharetratae Virginis arva coli?

crinibus insignem quis acuta cuspide Phoebum

instruat, Aoniam Marte movente lyram?

sunt tibi magna, puer, nimiumque potentia regna;

cur opus adfectas, ambitiose, novum?

(Amores I.1,5-14)

Aquí aparecen ampliados los dos puntos de partida señalados en los dos primeros versos, ideas arraigadas en la tradición de la doctrina poética de la época y que hubieron de extenderse y perdurar durante muchos siglos. Por un lado la superioridad del género épico sobre el amoroso, y por otro la necesidad de guardar armonía entre el tema, el género y el estilo. En este asunto Marlowe bebe de la ortodoxia más clásica en Ovidio, pero también de su subversión. Y asimila sobre todo, y traduce, unas jugosas y nada simplistas reflexiones sobre género y metro. El estilo asociado a cada uno de los géneros está directamente relacionado con el tipo de verso a usar:

Both verses were a like till love (men say)

Began to smile and tooke one foote away.

Rash boy, who gave thee power to change a line?

When in this workes first verse I trode aloft,

Love slackt my Muse, and made my numbers soft.

I have no mistresse, nor no favorit,

Being fittest matter for a wanton wit.

(I.1, vv. 7-9, 21-24)

par erat inferior versus--risisse Cupido

dicitur atque unum surripuisse pedem.

'Quis tibi, saeve puer, dedit hoc in carmina iuris?

cum bene surrexit versu nova pagina primo,

attenuat nervos proximus ille meos;

nec mihi materia est numeris levioribus apta,

aut puer aut longas compta puella comas.

(Amores,I.1,3-5 y 17-20)

Ovidio se refiere aquí, por supuesto, al pareado elegíaco que era el sofisticado vehículo para la elegía amorosa latina, compuesto de hexámetro y pentámetro (de ahí la "cojera" del pareado, con la que juega Ovidio). Como resalta Roma Gill en la introducción a su edición de la traducción de Marlowe (Gill. introd. pp.3-4), no resultaba fácil para el inglés de la época encontrar un equivalente a este metro, que requería un estilo para el cual el nuevo inglés moderno del siglo XVI todavía no había hallado la modulación adecuada. Por esta razón, Marlowe recurre al pareado heroico ("heroic couplet"), una forma ya establecida desde tiempos de Chaucer, y que en 
manos de otros poetas posteriores a Marlowe sería desarrollado en contextos más sofisticados -el propio Marlowe afinó mucho más esta forma en su Hero and Leander. Este tipo de metro, a pesar de haber alcanzado su madurez algo más tarde, corrió paralelo al decasílabo blanco como una de las formas más frecuentes en poesía inglesa, y durante el periodo entre 1640 y 1790 se convirtió en la forma dominante. Entre los más ilustres autores que usaron esta forma se cuentan Jonson y Donne. En esto es también Marlowe un adelantado, ya que a pesar de las limitaciones de su versión temprana de este pareado en la mayor parte de la traducción de los Amores, como ya se ha señalado arriba, en otras partes ya apunta la flexibilidad que habría de adquirir en el propio Hero and Leander, y sobre todo en poetas posteriores ${ }^{3}$. Dentro de este contexto de reflexiones sobre los géneros, y con el trasfondo del tema de los nuevos metros a adoptar, así como de la reforma y rehabilitación de metros ingleses tradicionales se sitúa por un lado, la canónica presencia de la protesta del poeta por intentar dedicarse al género épico, que ya era un topos en tiempos de Ovidio, con precedentes como Propercio ${ }^{4}$, pero sobre todo cabe destacar la reflexión sobre la adecuación entre género y metro. Ya vemos que Marlowe decide echar mano del pareado en esta traducción primeriza. Sin embargo, y en el empeño de tantos otros poetas de su tiempo por experimentar con nuevos metros y formas diferentes con vistas a construir un digno canon poético que imitase y emulase a los clásicos, Marlowe hubo de aprender y experimentar a raíz de una traducción posterior que el decasílabo blanco se prestaba mejor al tipo de flexibilidad que requería el inglés si pretendía emular la fluidez y la amplitud de los periodos poéticos de la mejor poesía latina. Dicho sea sin detrimento del camino que con no poco éxito emprendió el "heroic couplet". Un camino que le llevaría a un neoclasicismo que se podría definir como más ortodoxo, y que habría de culminar en Dryden y Pope, a pesar de que durante el intervalo entre su desarrollo a caballo entre los siglos XVI y XVII, y su agotamiento a finales del XVIII, hubo poetas que lo usaron para fines diversos de forma más que exitosa y sin esa especie de envaramiento que adoptó en los neoclásicos. El decasílabo blanco, o pentámetro blanco, sin embargo, tomó el camino del gran teatro isabelino -con Shakespeare por supuesto a la cabeza, pero sin olvidar al nutrido grupo de autores que se involucraron en el negocio dramático-hasta agotarse en Milton, que lo conduce y eleva hacia un latinismo más allá del cual resultará virtualmente imposible avanzar; de ahí el rechazo, entre otros, de T.S. Eliot, hacia esa tradición que sigue a Milton, y su intento de reevaluar el tono y la fluidez que el decasílabo blanco habían alcanzado, así como su frecuente proximidad a los esquemas tonales y rítmicos del habla normal de la época sin perder al mismo tiempo una dicción poética de muy variados registros.

\footnotetext{
${ }^{3}$ Véase Preminger \& Brogan 1993, p. 522: The transformation of the Chaucerian line into what Puttenham and Dryden called the 'heroic' line was evolutionary, spanning the 16th c. from ca. 1520 to 1600... But the imposition of the hierarchy of pauses on the loose couplet inherited from Chaucer was more dramatic, taking place almost explosively between 1590 and 1600, when numbers of English poets translated and adapted Latin poems in elegiac distichs... achieving or approximating a correspondence of couplet to distich... The immediate result of this structural imitation was the affixing onto English couplets of the Latin distich's hierarchy of pauses and of a correspondingly balanced rhetoric... English rhyme, which had the same closural power as the recurrent half-line pattern in the pentameter of the Latin distich, allowed the English measure, once it outgrew its dependence on the Latin model, to become much more flexible and thus absorb the modifications by which successive English poets from Donne to Crabbe transformed it into a medium of great expressive power.

${ }^{4}$ Sobre los antecedentes de la recusatio, y la original manera en que Ovidio hace uso de este topos véase McKeown 1987, vol. I, p. 12 ss.
} 
A la ortodoxia de las reflexiones de Ovidio sobre la jerarquía de los géneros y los

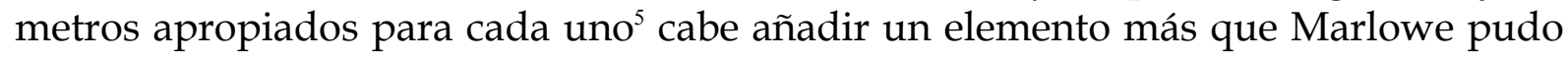
apreciar en Ovidio: el distanciamiento y la ironía, un cierto carácter subversivo y una diletante y provocadora rebeldía. Un ejemplo de esta ironía y distanciamiento irrespetuosos se halla en la primera elegía del segundo libro de los Amores, en los que frente a la puerta cerrada de su amante, Ovidio declara inútiles las armas de Júpiter, y los versos en alabanza de Aquiles, Héctor o Ajax. Dentro del contexto y del topos de la recusatio, Ovidio no sólo se autoacusa de lascivia ("wantonness"), sino que también admite que el tema restringe a su público-compuesto de jovencitas a las que describe como non frigida, y que sus débiles y despendolados versos han de ganar el rechazo de los más severos críticos (procul hinc, procul este, severae!). Todo el poema es una irónica y procaz parodia de los topos del verso elevado y sus fines, que Ovidio rechaza, adhiriéndose en su lugar a una empresa de escasa marcialidad, como es conseguir que su amada abra la puerta para consumar su unión. Obsérvese el anticlímax de los versos que describen el poder de la poesía, que hace retroceder al sol y la luna, así como el curso normal de los ríos, pero sobre todo hacen abrir hasta las puertas más sólidas. Ovidio insiste una vez más en la "blandura” y "ligereza" de sus versos-en oposición, se supone, a la virilidad y energía de los metros épicos, más del gusto de los severae (blanditias... / mollierunt duras lenia verba fores):

Verses deduce the horned bloudy moone

And call the sunnes white horses backe at noone.

Snakes leape by verse from caves of broken mountains

And turned streames run back-ward to their fountains.

Verses ope dores, and lockes put in the poast

Although of oake, to yeeld to verses boast.

(II.1, vv. 23-28)

Blanditias elegosque levis, mea tela, resumpsi;

Mollierunt duras leni verba fores.

Carmina sanguineae deducunt cornua lunae,

Et revocant niveos solis euntis equos;

Carmine dessiliunt abruptis faucibus angues,

Inque suos fontes versa recurrit aque.

Carminibus cessere fores, insertaque posti,

Quamvis robur erat, carmine victa sera est.

(Amores II.1,21-28)

Desde esta perspectiva resulta también interesante analizar la traducción de la primera elegía del tercer libro de los Amores, en la que el poeta, paseando en un bosque, se encuentra con las personificaciones de Elegia y Tragedia, la última de las cuales le reprocha que malgaste su juventud en la licenciosa práctica de la poesía amorosa, conminándole a que acometa empresas más altas en el género trágico. En este poema Ovidio viene a abundar más en el tema de convenciones de los géneros.

\footnotetext{
${ }^{5}$ Obsérvese que el cambio de metro en latín produce un pareado "cojo", flojo y suave, imperfecto y femenino, en contraposición a la energía, coherencia y rotundidad de la viril y marcial poesía épica: en este sentido la crítica feminista tendría mucho que decir, por no hablar de los nuevos "disabled studies".
} 
When will thy love be spent?

O Poet carelesse of thy argument.

Wine-bibbing banquets tell thy naughtinesse,

Each crosse waies corner doth as much expresse.

Oft some points at the prophet passing by,

And this is he whom fierce love burnes, they cry.

A laughing stock thou art to all the citty,

While without shame thou singst thy lewdnesse ditty.

Tis time to move grave things in lofty stile,

Long hast thou loyterd, greater workes compile.

The subject hides thy wit, means act resound,

This thou wilt say to be a worthy ground.

Thy muse hath played what may milde girles content,

And by those numbers is thy first youth spent.

Now give the Roman tragedy a name,

To fill my lawes thy wanton spirit frame.

(III.1, vv.15-30)

El poema muestra a Ovidio en su vertiente alegórica, de gusto más medieval. La debilidad y feminidad de la poesía amorosa, además de la cojera con que se acerca la alegoría de la Elegia, se contraponen aquí al agresivo avance de la personificación de la Tragedia. En el verso 10 (et pedibus vitium causa decoris erat), Ovidio declara, siguiendo con el juego de la analogía entre la cojera y el metro, que era justamente ese desequilibrio -en apariencia imperfecto- entre un pie y otro el origen del decoro y la belleza que muestra la Elegia. Se detecta aquí al Ovidio más subversivo, y quizá uno de los ejemplos en los que Marlowe pudo aprender de esta subversión creativa e inteligente de los topos clásicos, dado que en la descripción de las dos musas, a pesar de que los términos asociados con la Elegia son en principio negativos (cojera, superficialidad, lascivia), éstos en contraposición con los de la tragedia (igual que con anterioridad se ha hecho con el género épico) resultan positivos. La Tragedia aparece ante el lector con un rostro violento y a grandes zancadas, con mirada torva, dando la imagen de una suerte de gigante torpe y mal encarado, en comparación con la inusual gracia, sensualidad y belleza de los pasos desiguales de la Elegia, envuelta en transparentes y vaporosos ropajes. De esta forma, Ovidio subvierte con ingenio la jerarquía de los géneros, y sustituye la anterior tradición de la recusatio y su marcado tono de disculpa por una irónica burla de la figura que alegoriza a la musa trágica, supuestamente más elevada y digna. Estamos ante un caso de frescura poética en el más amplio y profundo de los sentidos. Esta mezcla compleja, este retorcer y sofisticar los temas anteriores sería la enseñanza de la que Marlowe sacaría unos magníficos réditos al cabo de su carrera como poeta y dramaturgo.

Elegia came with haires perfumed sweete,

And one, I thinke, was longer, of her feete.

A decent forme, thinne robe, a lovers looke,

By her footes blemish greater grace she tooke.

Then with huge steps came violent Tragedie,

Sterne was her front, her cloake on ground did lie.

Her left hand held abroad a regal sceptre, 
The Lydian buskin in fit paces kept her.

$$
\text { (III.1, vv. 7-14) }
$$

venit odoratos Elegia nexa capiillos, et, puto, pes illi longior alter erat.

Forma decens, vestis tenuissima, vultus amantis,

Et pedibus vitium causa decoris erat.

Venit et ingenti violenta Tragoedia passu:

Fronte comae torva, palla iacebat humi;

Laeva manus sceptrum late regale movebat,

Lydius alta pedum vincla cothurnus erat.

$$
\text { (III.1,7-14) }
$$

La elección de Ovidio y de sus elegías amorosas propició así en Marlowe la reflexión no sólo acerca del establecimiento de un canon, y las formas poéticas asociadas con los diferentes géneros, sino también sobre estrategias para salir de los caminos trillados, sobre cómo diferir de lo anterior construyendo sobre ello al mismo tiempo, de forma creativa y sin someterse de manera servil. En este sentido, y además de las reflexiones sobre género y forma poética, en otra de las elegías (I.15, q.v.), Marlowe pudo encontrar una jugosa lista de autores en el marco de una reflexión de Ovidio sobre la inmortalidad de la poesía, un tema tan presente no sólo en Ovidio, sino en otros autores centrales del canon latino -de forma notable en Horacio, como es sabido.

Tanto en Ovidio como en Lucano, además, Marlowe pudo encontrar una actitud de distanciamiento con respecto a la idea de imperio y el optimismo histórico con él asociado, así como los procedimientos retóricos y estilísticos sobre los que articular el caos que emerge del fracaso de un proyecto a la vez moral, social y político, de la desintegración de la República y la decadencia del Imperio6. Este tono y este trasfondo se unen a la variedad de registros que confluyen en la obra poética y dramática de Christopher Marlowe.

Hay dos aspectos que cabe comparar a la hora de valorar la decisión de Marlowe de llevar a cabo una traducción de los Amores de Ovidio y sacar de ella consecuencias para la posterior carrera literaria de Marlowe y sus avatares críticos, aspectos relacionados de una forma $\mathrm{u}$ otra con las diferencias entre géneros literarios y su posición dentro del canon. Son aspectos éstos que merecen un análisis más profundo, pero que por motivos de tiempo y espacio sólo esbozaré brevemente. Uno de estos aspectos, ya en parte mencionado, es la progresión de la práctica de géneros a lo largo de la carrera de un poeta, establecidos por una antigua tradición dentro del canon. Esta especie de cursus honorum del poeta que aspira a consolidar su prestigio incluye partir de géneros menores (elegía amorosa, poesía lírica en general, poesía pastoral) hasta ir perfeccionando la práctica poética hacia los géneros más elevados, principalmente las musas trágica y épica. En este sentido, Marlowe despliega su particular progresión, cuyos primeros pasos, repito, están marcados por la práctica de la

\footnotetext{
${ }^{6}$ Resulta sugerente imaginar la agresiva figura de la Tragedia, severa y pesada, como un emblema de la idea que Ovidio tendría del proyecto imperial de Augusto, de su campaña de moralidad pública, que respondía, por otra parte, a los propios excesos de la familia del emperador. No se olvide que Ovidio rechazó perseguir una carrera política, y que la vida fácil -y para algunos disoluta- de un literato diletante le resultaba más atractiva que la seriedad de las funciones públicas asociadas a la alta burocracia imperial.
} 
traducción, principalmente de Ovidio y Lucano. Como se sabe, la carrera y la vida de Marlowe quedan truncadas, como truncada se halla en parte la que tradicionalmente se ha considerado su obra principal, el Fausto, una obra articulada sobre fragmentos brillantes, en la que se advierte la mano de más de un autor. Lo interesante del canon moderno en cuya base se halla Marlowe es que la épica queda -aunque no del tododesplazada e integrada -también en parte- dentro de la escena isabelina.

Otro paralelismo interesante entre las carreras y los textos de Marlowe y Ovidio, contemplados cada uno de ellos dentro del contexto de la educación y el ambiente literario e intelectual de sus respectivas épocas, es el que concierne a los temas de oralidad y textualidad. La oralidad contemplada sobre todo desde la relación existente entre texto poético escrito y declamación. Con anterioridad he señalado lo acertado de la traducción de Lucano de Marlowe. Pues bien, este autor era considerado por Quintiliano más apropiado para la declamación que para la lectura, más digno de imitación por los oradores que por los propios poetas. ${ }^{7}$. Igualmente, la crítica de Ovidio ha señalado con frecuencia el carácter declamatorio de mucha de su poesía, y más en concreto de los Amores ${ }^{8}$. Bajo esta perspectiva, resulta lógico considerar estas dos traducciones de Marlowe como una excelente elección para ejercitar una voz poética que habría de desplegarse de lleno en los abigarrados escenarios londinenses. Cabe destacar igualmente la flexibilidad y movilidad entre los diferentes géneros, y cómo si en la antigüedad el teatro y la declamación pública servían para encauzar este aspecto oral de la producción poética, en el Renacimiento la escena isabelina ofrecía a los autores una base similar para desarrollar estas cualidades específicas. Como base de este aspecto en la poesía, cabe destacar también la educación en ejercicios de retórica y declamación no sólo en la antigüedad, sino también los que tenían lugar en el renacimiento. A la luz de estas consideraciones, la particular progresión poética de Marlowe adquiere una coherencia ejemplar.

La escena isabelina se convierte en un fascinante laboratorio donde se experimenta con diferentes tipos de discursos y de convenciones genéricas. Y esto se hace de cara al público y la sociedad londinense. Los autores colaboran entre sí, difuminando con frecuencia la noción tradicional de autor, y se juegan la vida, a veces casi literalmente, en la composición de sus obras, que se estrenan y consumen a un ritmo frenético. La novedad de este medio, de su público, y de sus esquemas productivos facilitaban la creatividad de los autores, así como el "fusilamiento", con frecuencia atropellado y sistemático, de géneros y textos tanto anteriores como contemporáneos, autóctonos y también extranjeros. Esto explica la inmediatez y fugacidad de la escena, su dinamismo, frente a textos más permanentes, como los poemas narrativos o las novelas en prosa. En este sentido no olvidemos que los textos que nos han llegado han sido generalmente los que gozaron de más éxito y favor del público, y que por tanto los editores consideraron merecedores de imprimir, entre otras cosas, por el beneficio económico que les reportarían. La escena isabelina se puede contemplar como una especie de antecedente de la novela en tanto que cajón de sastre de estilos, recursos y personajes, amalgama resultante de la reorganización de las tradiciones anteriores. Es igualmente significativo que el teatro isabelino, como espectáculo pú-

\footnotetext{
${ }^{7}$ Quintiliano, Inst. Or. X.1,90: Lucanus ardens et concitatus et sententiis clarissimus, et, ut dicam quod sentio, magis oratoribus quam poetis imitandus.

${ }^{8}$ Véase McKeown 1987 vol. I, pp.34, 63, 65, 67-69 y 71.
} 
blico y popular, venga en parte a llenar el espacio que antes ocupaban los espectáculos sacros desaparecidos tras el advenimiento de la Reforma. De ahí también lo que de estos personajes estereotipados toman autores como el propio Marlowe: parte de la trama bufa de Fausto, y que a juicio de la crítica encaja menos con la pluma y el proyecto "serio" de Marlowe, proviene precisamente de los demonios de estos espectáculos populares".

Esta complejidad de elementos que vienen a componer y entre los cuales se incardina el teatro isabelino es paradigmática de la fragmentación y reagrupación renacentista, que también tiene lugar en otros géneros de esta época. Marlowe es un excelente ejemplo de esta reordenación. Más allá del marco conceptual del género teatral -no ya, siquiera, entre comedia y tragedia- la variedad de tonos, situaciones y personajes -incluído también el horizonte de expectativas del público isabelino- resulta compleja y -a ojos de un público y una crítica posterior, acostumbrados a obras más unitarias- sorprendente. En este sentido, la fragmentación de discursos que tiene lugar en el tránsito del siglo XV al XVI, y de éste al XVII tiene como resultado una nueva y contínua restructuración dinámica que da lugar a la escena comercial inglesa. El declive de los fenómenos dramáticos de carácter popular asociados al catolicismo medieval (morality plays, mystery plays, etc.), la creación de una nueva audiencia urbana en Londres, y el carácter mercantil -incluso competitivo- contribuyen a crear el perfil de la escena inglesa de este periodo. Al mismo tiempo, cierto alejamiento de los círculos académicos -téngase en cuenta el origen universitario de muchos de estos autores, pero también la falta de una educación universitaria formal en el propio Shakespeare- y aristocráticos (dicho dicho sea esto hasta cierto punto y con matices, ya que las relaciones entre las compañías y la corte eran con frecuencia fluidas: quizá otra peculiaridad del momento sea el hecho de que el mismo género compartía audiencias tan diferentes, y divergentes, como la corte y las muchedumbres de Londres que acudían casi a diario al teatro) le confieren además esa cierta libertad, una amplia banda de oscilación que le permitía incluir convenciones genéricas provenientes de ambitos muy diversos: poesía pastoral, poemas épicos, reflexiones morales, teatro sacro, además de comedia y tragedia, romances caballerescos, y una larga lista. Una parte considerable del teatro isabelino, y el Doctor Faustus de Marlowe en concreto -como viene demostrando la crítica últimamente- desafían no ya las fronteras entre géneros, sino el concepto mismo de autor individual, ya que en estas obras no era infrecuente la invervención de varias manos para quitar, añadir o modificar, en muchas ocasiones por razones de mercado.

En relación a Marlowe, su Doctor Faustus presenta como pocas este carácter fragmentario: ecos de "morality plays", personajes bufos que evocan a los demonios y los personajes de los ciclos de obras pseudosacras anteriores, el verso bombástico de Marlowe, forjado en sus traducciones de Lucano, la recusatio o el rechazo del género épico al comienzo de la obra, para adentrarse en los avatares antiépicos de la ambiciòn desmedida y frustrada del itinerario vital del doctor Fausto, la posible presencia de otro autor al menos, la existencia de dos textos de carácter diferente, la con-

\footnotetext{
${ }^{9}$ La introducción de David Ormerod y Christopher Mortan a su edición del llamado "Texto A" de Doctor Faustus ofrece un buen resumen de la polémica acerca de los añadidos que se consideran ajenos a la pluma de Marlowe, así como al tema de los diferentes autores y tramas entretejidas con el supuesto texto original de Marlowe (supuestamente el "Texto A", en oposición a la versión más larga que recoge los añadidos por otros autores).
} 
troversia sobre qué parte corresponde a la autoría de Marlowe y cuál a otro/s autor/es, etc. En este sentido, el Doctor Faustus es emblemática de la poliédrica y fragmentaria naturaleza de este nuevo teatro. Pero también su destino posterior, como generador de otros textos (Goethe, Thomas Mann, etc.) convierten al Fausto de Marlowe en una obra-eje, integradora de textos, géneros y discursos anteriores, y a la vez impulsora de nuevos textos.

Tanto para Ovidio, cuando rechaza el género épico a favor del elegíaco, como para Marlowe, cuando rechaza los grandes temas militares de la poesía heróica y el formato del poema épico a favor del tema más restringido de Fausto como individuo recluído en su estudio, en términos de género y de sujetos, este menos es más:

Not marching in the fields of Thracimene,

Where Mars did mate the Carthaginians,

Nor sporting in the dalliance of love

In courts of kings, where state is overturned,

Nor in the pomp of proud audacious deeds,

Intends our muse to vaunt his heavenly verse:

Only this -Gentlemen- we must perform

The form of Faustus' fortunes, good or bad.

$\cdots$

And this the man that in his study sits.

(Faustus, vv. 1-28)

Partiendo de la recusatio de la primera elegía de Ovidio, y siguiendo su ejemplo de compleja ironía y de distanciamiento, al tiempo que toma lo más valioso de los esquemas conceptuales de géneros anteriores, en Dr Faustus Marlowe reúne dentro del género dramático el clásico rechazo del discurso épico (rechazando también de paso el género amoroso) con la propia dicción épica que había aprendido en Lucano. Con estas herramientas y materiales Marlowe construye sobre la escena lo que constituye la raíz de la épica moderna: el viaje a la conciencia y las contradicciones de la subjetividad individual, la tragedia de Fausto, el científico e intelectual de ambición, que había de convertirse en emblema del hombre moderno en la tradición romántica. Precisamente si Marlowe convierte a su Faustus en una obra-eje dentro del canon occidental, fue porque en ella combinó de forma heterodoxa y relevante las convenciones y las desviaciones de los mejores poetas que le precedieron, y en los que se educó por medio de la práctica de la traducción. El resultado fue una obra que lanzó estas convenciones anteriores hacia el futuro, transformadas e integradas dentro de un género renovado, la escena isabelina, que habría de ofrecer a uno de los grandes del canon, Shakespeare, y con una temática renovada: la odisea de la contradictoria subjetividad individual. Podemos afirmar, con Harold Bloom, que Shakespeare creó la idea moderna de individuo, pero Marlowe se anticipó de forma lúcida al combinar géneros y formas para dar sustancia verbal a la dirección que tomaría el canon occidental durante los siguientes siglos. 


\section{Bibliografía}

Butler, H.E., trans. 1922. The Institutio Oratoria of Quintilian, vol. IV. Books X-XII. Ed. bilingüe. The Loeb Classical Library. Cambridge, Mass. \& London, England: Harvard University Press.

Gill, Roma, ed. 1997. The Complete Works of Christopher Marlowe. Vol. I. All Ovids Elegies, Lucans First Booke, Dido Queene of Carthage, Hero and Leander. Oxford: Clarendon Press.

McKeown, J.C., ed. \& introd. 1987. Ovid: Amores. Text, Prolegomena, and Commentary. 4 vols. Vol. I Text and Prolegomena. Liverpool: Francis Cairns Publications.

Ormerod, David, \& Christopher Wortham, eds. 1989. Christopher Marlowe. Dr Faustus: The A-Text. Nedlands, W.A.: University of Western Australia Press.

Preminger, Alex, \& T.V.F. Brogan, eds. 1993. The New Princeton Encyclopedia of Poetry and Poetics, Princeton, NJ: Princeton University Press.

Woods, Susanne. 1985. Natural Emphasis. English Versification from Chaucer to Dryden, San Marino: The Huntington Library. 